\title{
Polymerase chain reaction and immunohistochemistry frequently detect occult melanoma cells in regional lymph nodes of melanoma patients
}

\author{
N Hatta, M Takata, K Takehara, K Ohara
}

\begin{abstract}
Aims-To evaluate immunohistochemistry and reverse transcriptase polymerase chain reaction (RT-PCR) for melanoma associated antigens (MAA) in detecting occult melanoma cells in lymph nodes which were missed on routine pathology. Methods-Occult melanoma cells were sought in 436 lymph nodes from 32 patients with cutaneous melanoma of the lower extremities by immunohistochemistry using the melanoma specific antibody HMB-45. The detection sensitivity of routine histology, immunohistochemistry, and RT-PCR was also compared in 23 lymph node samples from six patients. Results-Immunohistochemistry showed that 15 of 24 patients $(62.5 \%)$ who had no detectable metastasis by routine histology had at least one lymph node containing HMB-45 positive cells, mostly seen singly in the medullary sinus. No difference was found in known clinicopathological prognostic factors and recurrence rates between the two groups of patients with and without occult HMB-45 positive cells. RT-PCR analyses showed that the nested PCR for tyrosinase was more sensitive than a combination of single round PCR for five different MAA, including tyrosinase, MART-1/Melan A, Pmel-17, TRP-1, and TRP-2, detecting tyrosinase $M R N A$ in six nodes which were negative by HMB-45 immunohistochemistry.

Conclusions-Immunohistochemistry + RT-PCR is more sensitive than routine histology in detecting occult melanoma cells in lymph nodes. The nested PCR for tyrosinase should be used in future studies investigating the prognostic significance of such lymph node micrometastases. (F Clin Pathol 1998;51:597-601)
\end{abstract}

Keywords: melanoma; metastasis; tyrosinase; immunohistochemistry

It is well recognised that the presence or absence of lymph node metastases in patients with malignant melanoma is the most powerful factor predicting survival. ${ }^{1}$ Accurate staging by the examination of resected lymph node specimens allows patients at risk to be offered adjuvant immunotherapy. ${ }^{2}$ Furthermore, the recent development of preoperative or intraoperative lymphatic mapping and sentinel node biopsy for detecting the presence or absence of metas- tases in the first draining lymph node enables selection of those patients most likely to benefit from elective lymph node dissection. ${ }^{3}$ Although in the past, lymph node specimens were examined by standard pathology techniques with haematoxylin-eosin staining, recent investigations have shown that immunohistochemistry, ${ }^{34}$ cell culture, ${ }^{5}$ and reverse transcription coupled with polymerase chain reaction (RT-PCR) ${ }^{67}$ are more sensitive at detecting occult melanoma cells. In the present study, we examined the frequency of occult melanoma cells in the lymph nodes of patients with malignant melanoma, and compared the detection sensitivity of immunohistochemistry and several different RT-PCR assays.

\section{Methods}

LYMPH NODE SAMPLES

We examined 436 inguinal lymph nodes from 32 patients with cutaneous melanoma of the lower extremities (two superficial spreading melanomas, eight nodular melanomas, and 22 acral lentiginous melanomas) who underwent inguinal lymph node dissection at the Kanazawa University Hospital and the Toranomon Hospital. All the patients had a primary tumour more than $1.5 \mathrm{~mm}$ in thickness, except for two who had tumours 1.0 and $1.3 \mathrm{~mm}$ thick. Eight patients had lymphadenopathy before treatment, while another 24 had clinically impalpable nodes. Each specimen was fixed in $10 \%$ neutral buffered paraformaldehyde and embedded in paraffin. The portions of 23 lymph nodes from six patients were immediately frozen in liquid nitrogen for mRNA extraction. As negative controls for immunohistochemistry and RT-PCR, we used 44 paraffin embedded and 10 frozen lymph node samples from 24 patients with nonmelanoma skin cancers and inflammatory skin diseases.

IMMUNOHISTOCHEMISTRY

Four micrometre thick paraffin sections were deparaffinised, rehydrated, and stained with melanoma associated monoclonal antibody HMB-45 (Dako, Carpinteria, California, USA) using the alkaline phosphatase conjugated streptoavidin-biotin system (Histofine Kit; Nichirei, Tokyo, Japan). The reaction products were visualised using new fuchsin as a chromogen. The sections were counterstained with haematoxylin. As negative controls, primary antibody was replaced with non-immune mouse immunoglobulin. 

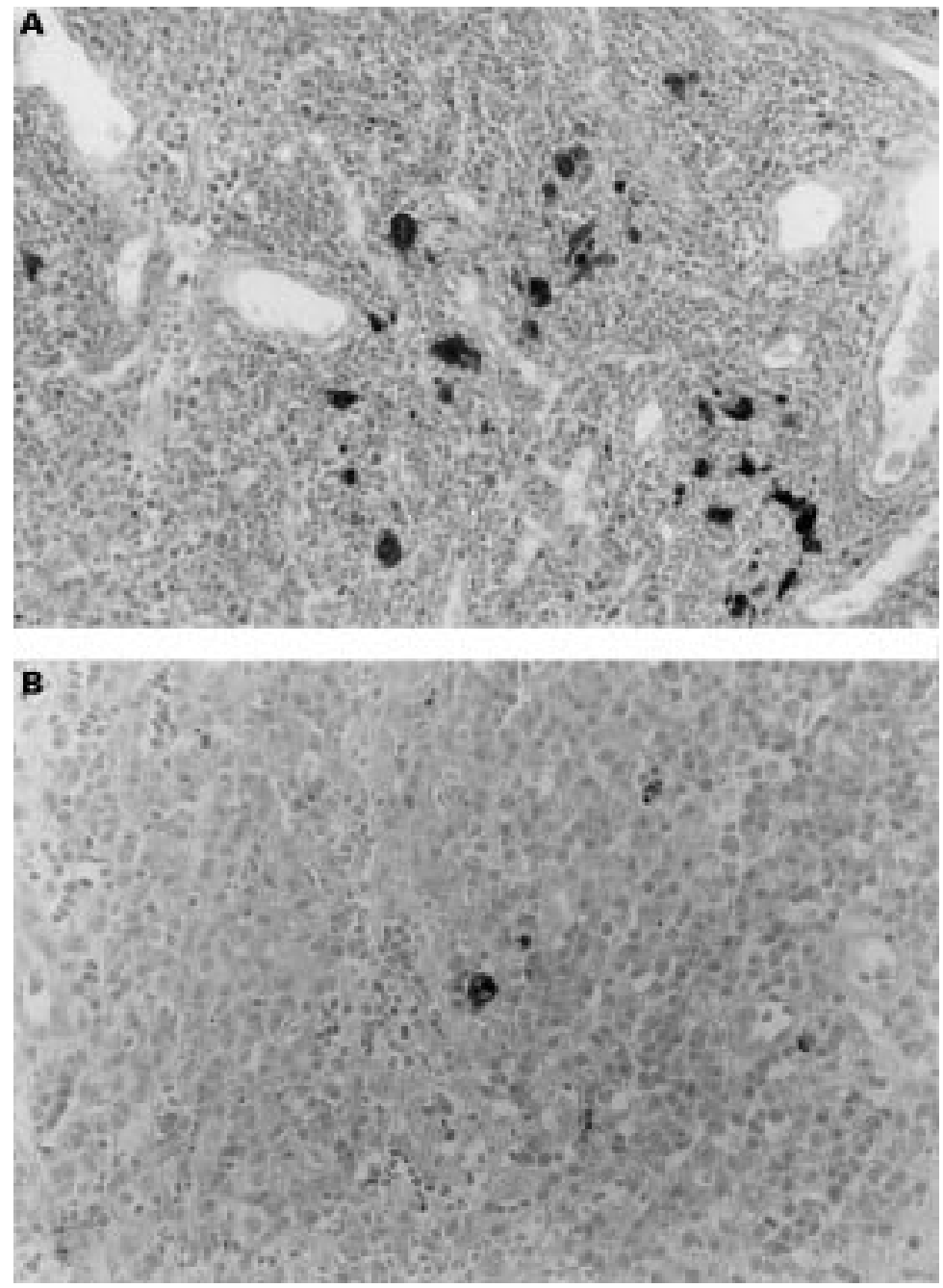

Figure 1 An example of HMB-45 positive tumour cells in a histologically "node negative"lymph node. HMB-45 positive cells were seen in small groups $(A)$, or singly in medullary sinus (B) (biotin-streptavidin-alkaline phosphatase method, $\times 130$ ).

RNA PREPARATION

Total cellular RNA was isolated by the acid guanidinium thiocyanate procedure (Isogen; Nippon Gene, Tokyo, Japan) from frozen lymph node samples. Briefly, the cells were lysed in $1 \mathrm{ml}$ of Isogen reagent by homogenisation and placed on ice for five minutes; 200 $\mu l$ of chloroform were added, mixed vigorously for 15 seconds, placed on ice for five minutes, and then centrifuged at $12000 \mathrm{~g}$ at $4^{\circ} \mathrm{C}$ for 15

Table 1 Clinicopathologic characteristics of histologically node negative patients with and without HMB-45 positive tumour cells in lymph nodes

\begin{tabular}{llll}
\hline & $\begin{array}{l}\text { HMB-45 } \\
\text { negative } \\
(n=9)\end{array}$ & $\begin{array}{l}\text { HMB-45 } \\
\text { positive } \\
(n=15)\end{array}$ & p Value* \\
\hline $\begin{array}{l}\text { Variable } \\
\text { Breslow's thickness }\end{array}$ & 3 & 10 & 0.11 \\
$\quad<4.0 \mathrm{~mm}$ & 6 & 5 & \\
$\quad \begin{array}{l}\text { Level } \\
\text { III }\end{array}$ & 0 & 3 & \\
IV & 0 & 12 & 0.17 \\
V & 1 & 0 & \\
Ulceration & & & \\
$\quad$ Negative & 5 & 8 & 0.92 \\
$\quad$ Positive & 4 & 7 & \\
Recurrence & & & \\
$\quad$ Negative & 8 & 12 & 0.57 \\
Positive & 1 & 3 & \\
\hline
\end{tabular}

*The $\mathrm{p}$ values of the $\chi^{2}$ test between the two groups. minutes. The upper phase was transferred into a new Eppendorf tube, $500 \mu$ of isopropanol added, and the RNA precipitated at $4^{\circ} \mathrm{C}$ for 10 minutes. The sample was washed with $70 \%$ ethanol, dried, and resuspended in $40 \mu \mathrm{l}$ of RNAse-free water.

OLIGONUCLEOTIDE PRIMERS

Oligonucleotide 5' and 3' primers for individual genes were synthesised as follows ${ }^{8}{ }^{9}$ : tyrosinase, 5'-TTGGCAGATTGTCTGTAGCC-3' and 3'-AGGCATTGTGCATGCTGCTT-5'; tyrosinase (nested primers), 5'-GTCTTTATGCAATGGAACGC-3' and 3'-GCTATCCCAGTAAGTGGACT-5'; TRP-1, 5'-AGAGATGATCGGGAGGTCTG-3' and 3'-CTGTGCCATGTGAGAAAAGC-5; TRP-2，5'-GAGGTGCGAGCCGACACAAG-3' and 3'-CGGTGCAGGTAACAAATGC-5'; Pmel 17, 5'-TGGACCTTGCCCATCTGGCTCTTGG-3' and 3'-TGCCCATCTGTGGTGCCTGGAACTG-5'; MART-1/Melan-A，5'-CACGGCCACTCTTACACCAC-3' and 3'-GGAGCATTGGGAACCACAGG-5'. The RT-PCR products of tyrosinase, tyrosinase with nested PCR, TRP-1, TRP-2, Pmel 17, and MART-1/ Melan-A were 284, 207, 425, 476, 533, and 252 $\mathrm{bp}$, respectively.

\section{RT-PCR ANALYSIS}

First strand cDNA synthesis was performed using an RNA PCR Kit (Takara, Otsu, Japan). Briefly, the RT mixture consisted of $1 \times$ RNA PCR buffer, $5 \mathrm{mM}$ of $\mathrm{MgCl}_{2}, 1 \mathrm{mM}$ of each NTP, $1 \mathrm{U} / \mu \mathrm{l}$ of RNAse inhibitor, $0.125 \mu \mathrm{M}$ of oligo dT primer, and $0.25 \mathrm{U} / \mu \mathrm{l}$ of avian myeloblastosis virus reverse transcriptase. One microgram of RNA and water was added to the RT mixture to a final volume of $20 \mu \mathrm{l}$, and the reaction mixture incubated at $55^{\circ} \mathrm{C}$ for 30 minutes, at $99^{\circ} \mathrm{C}$ for five minutes, and then on ice for five minutes. The quality of cDNA synthesis was monitored by using $\beta$ actin primers, 5'-CTGTCTGGCGGCACCACCAT-3' and 3'-GCAACTAAGTCATAGTCCGC-5'. ${ }^{10}$

The PCR mixture consisted of $1 \times$ thermophilic reaction buffer (Promega, Madison, Wisconsin, USA), $200 \mu \mathrm{M}$ of each dNTP, 2 $\mathrm{mM} \mathrm{MgCl}, 100 \mathrm{pM}$ of each oligonucleotide primer, and 5 units of Taq DNA polymerase and cDNA. The PCR mixture was incubated in a thermal cycler at $95^{\circ} \mathrm{C}$ for five minutes for one cycle; $95^{\circ} \mathrm{C}$ for one minute, $55^{\circ} \mathrm{C}$ for one minute, and $72^{\circ} \mathrm{C}$ for one minute for 35 cycles; and $72^{\circ} \mathrm{C}$ for 10 minutes. For reamplification with tyrosinase nested primers, $2.5 \mu \mathrm{l}$ of the first PCR products were subjected to an additional cycle of $96^{\circ} \mathrm{C}$ for three minutes followed by 25 cycles of $94^{\circ} \mathrm{C}$ for one minute, $60^{\circ} \mathrm{C}$ for 45 seconds, and $72^{\circ} \mathrm{C}$ for 30 seconds with an $\mathrm{MgCl}_{2}$ concentration of $2.25 \mathrm{mM}$. The amplified DNA samples were run on a $1.5 \%$ agarose gel, and bands were visualised with ethidium bromide.

\section{STATISTICAL ANALYSIS}

Categoric data were compared by the $\chi^{2}$ test. Probability $(p)$ values $<0.05$ were considered statistically significant. All analyses were 


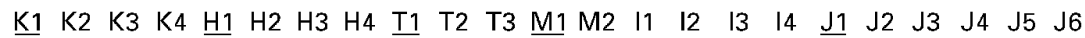
Histology $\square \square \square \square \square \square \square \square \square \square \square \square \square \square \square \square \square \square \square \square \square \square$

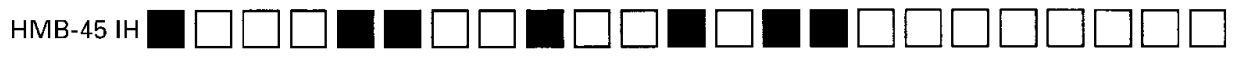
Tyrosinase
(Single PCR)
Sy $\square \square \square \square \square \square \square \square \square \square \square \square \square \square \square \square \square \square \square \square$

Tyrosinase

(Nested PCR)

MART-1/Melan-A

Pmel-17 mana $\square$

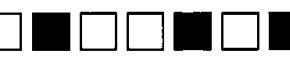

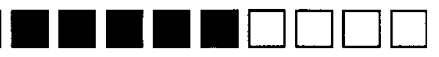
TRP-1 $\square \square \square \square \square \square \square \square \square \square \square \square \square \square \square \square \square \square \square \square \square \square$ TRP-2

Figure 2 Results of histopathological, immunohistochemical, and reverse transcriptase polymerase chain reaction (RT-PCR) analyses in 23 lymph nodes from six patients. Lymph node samples are numbered proximal to distal from the primary site. $\square$, negative, $\square$, positive. Possible sentinel nodes are underlined.

performed using StatView J version 4.5 software (Abacus Concepts Inc, Berkeley, California, USA).

\section{Results}

Routine pathological examination of haematoxylin-eosin stained sections of 436 inguinal lymph nodes from 32 patients showed metastases in 17 lymph nodes $(3.9 \%)$ from eight patients $(25 \%)$. All these eight patients had palpable nodes before resection. The 331 lymph nodes from the remaining 24 patients were negative by routine histology. However, immunohistochemistry for melanoma associated antigen HMB-45 showed that 47 lymph nodes (14\%) from $15(62.5 \%)$ of these "node negative" patients contained tumour cells stained with HMB-45 antibody. The HMB-45 positive cells were seen in small clusters (five to 20 cells) in three lymph nodes from two patients, whereas in the remaining 44 nodes, HMB-45 positive cells were mostly present singly through the section (fig $1 \mathrm{~A}$ and $1 \mathrm{~B}$ ). These HMB-45 positive cells were located
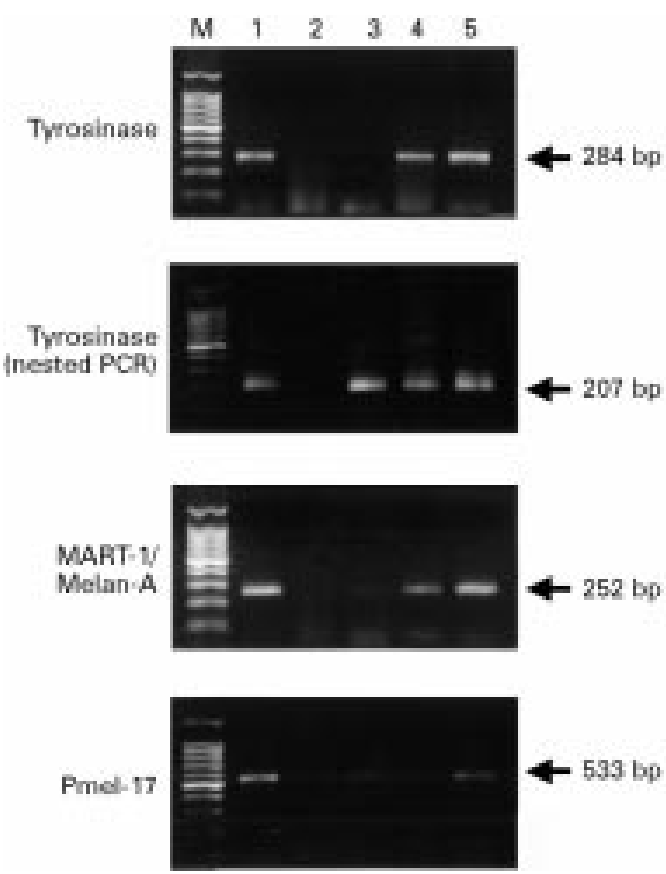

mainly in the medullary sinus of lymph nodes, although some were also seen in the marginal sinus in a few nodes. None of the 44 lymph nodes from patients with non-melanoma skin cancers and inflammatory skin diseases contained HMB- 45 positive cells.

We examined several prognostic factors including Breslow's tumour thickness, Clark's level of invasion, and ulceration of the primary tumour for possible correlation with the presence of occult melanoma cells revealed by HMB-45 antibody (table 1). No association was found between the HMB-45 immunostaining positivity and the clinicopathological characteristics. There was no difference in recurrence rate between occult tumour cell positive and negative patient groups. The follow up periods of these 24 patients ranged from 12 to 96 months (median 45 months).

We then carried out comparative histopathological, immunohistochemical, and RT-PCR analyses in 23 fresh frozen lymph node samples obtained from six patients. Figure 2 shows the
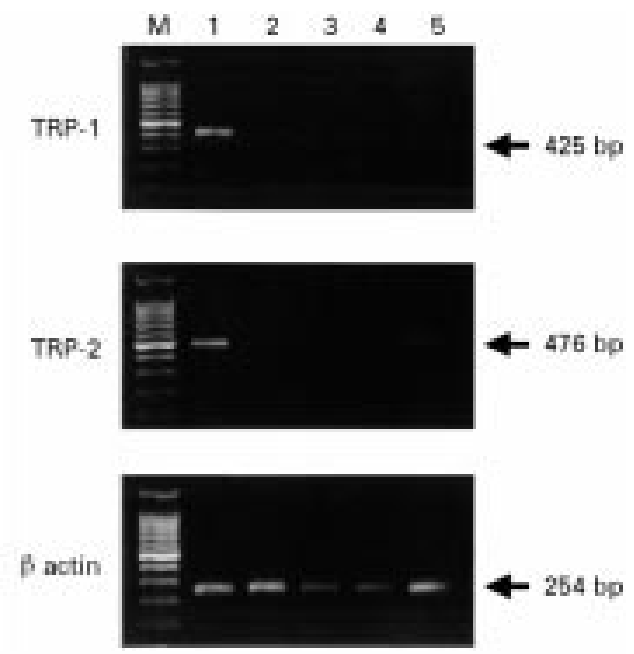

$533 b p$

Figure 3 Reverse transcriptase polymerase chain reaction (RT-PCR) analysis of the melanoma associated antigen $m R N A$ expression in a representative case. Lanes 1, 2, 3, 4, and 5 correspond to K1, H3, T1, I1, and $f 1$ in fig 2, respectively. $M$, 100 bp size marker. 
details of histopathological, immunohistochemical, and RT-PCR analyses in individual nodes and the representative RT-PCR gels are shown in fig 3 . Four of 23 lymph nodes (17\%) contained histologically proven metastases. Immunohistochemistry for HMB-45 revealed positive cells in seven lymph nodes (30\%), including three histologically negative nodes. With a single round PCR for five melanoma associated antigens, mRNA expression of tyrosinase was detected in five nodes $(22 \%)$, of MART-1/Melan A in five (22\%), of Pmel-17 in six $(26 \%)$, of TRP-1 in one (4\%), and of TRP-2 in two $(9 \%)$. Tyrosinase transcripts were detected in 14 lymph nodes $(61 \%)$ by using nested primers. No mRNA expression was detected for any MAA in 10 lymph nodes obtained from non-melanoma patients. All the seven nodes that were positive by immunohistochemistry were positive for tyrosinase mRNA expression, as revealed by nested PCR. Nested PCR for tyrosinase detected mRNA expression in an additional seven nodes. Nine nodes that were negative for tyrosinase by nested PCR were also negative by immunohistochemistry and routine histology. By contrast, the results from single round PCR for MAA were rather inconsistent: RT-PCR for MART-1/Melan A, Pmel-17, TRP-1, and TRP-2 was sometimes negative even in the histologically positive nodes. In two lymph nodes shown to contain occult melanoma cells by immunohistochemistry, mRNA expression was not detected for any of the MAA by single round PCR.

\section{Discussion}

The monoclonal antibody HMB-45 is highly specific to melanoma cells and junctional nevomelanocytes, and does not react with normal adult melanocytes or a range of normal tissues including lymph nodes, ${ }^{112}$ although sweat gland cells and breast lobules and ducts, as well as a very small subset of breast carcinoma cells, can occasionally show positive HMB-45 immunoreactivity. ${ }^{13}$ We did not detect any HMB-45 immunoreactivity in 44 control lymph nodes from cases other than melanoma. Thus the HMB-45 positive cells we observed in the lymph nodes from melanoma patients are thought to be occult melanoma cells draining from the primary tumour site.

We found such HMB-45 positive occult melanoma cells in at least one lymph node in 15 of 24 patients $(62.5 \%)$ who had no histologically documented lymph node metastases. These occult melanoma cells were mostly dispersed singly within the medullary sinus. Cochran et al, ${ }^{4}$ who examined 2227 lymph nodes from 100 node negative patients by immunohistochemistry for S-100 protein and $\mathrm{NK} 1 / \mathrm{C} 3$, found that 16 nodes from 14 patients $(14 \%)$ had detectable melanoma cells. The much higher incidence of occult melanoma cells detected in our study probably results from the use of the highly specific HMB-45 antibody for immunohistochemical staining. Because the S-100 protein antibody used by Cochran et al reacts with several normal resident cells in lymph nodes, such as interdigitating reticulum cells, sinus macrophages, and
Schwann cells in node associated nerves, ${ }^{4}$ it is very likely that the single occult melanoma cells we observed in this study using the HMB-45 antibody would not have been distinguished from S-100 positive normal lymph node constituents. We observed a small cluster of melanoma cells which we suspect would have been recognised as micrometastases by S-100 protein staining in only two of 24 patients $(8 \%)$. The difference in the patient population between the two studies might also have affected the results, as $38 \%$ of patients examined by Cochran et al had a primary tumour less than $1.5 \mathrm{~mm}$ thick.

The sensitivity of detection of such occult melanoma cells in the lymph node seems to be further increased by the use of RT-PCR for MAA, especially by nested PCR for tyrosinase. As reported by Van-der-Velde-Zimmermann et $a l,{ }^{7}$ we detected tyrosinase mRNA expression not only in all HMB-45 positive lymph nodes, but also in seven nodes in which HMB-45 immunoreactivity were entirely negative. The higher sensitivity of RT-PCR compared with HMB-45 immunostaining in detecting occult melanoma cells is not surprising, because the sensitivity for identifying melanoma cells against a background of normal cells is estimated to be approximately $1: 10^{5}$ by immunohistochemistry ${ }^{3}$ and $1: 10^{6}$ by RT-PCR for tyrosinase..$^{6-8}$ Furthermore, one can examine only a limited number of sections (normally representing $1 / 1000$ of the lymph node tissue) by immunohistochemistry, whereas mRNA is extracted for RT-PCR analysis from a substantial part of the lymph node. ${ }^{6}$ Recent studies by Sarantou et al and Hoon et al emphasised that the multimarker MAA RT-PCR analysis is more reliable and sensitive than a single molecular assay for the detection of melanoma cells. ${ }^{14}$ This seems reasonable because tumours such as melanoma are heterogeneous in gene expression, particularly in the advanced stages of tumour progression. This study also showed that the combination of tyrosinase, MART-1/Melan A, Pmel-17, TRP-1, and TRP-2 increased the detection sensitivity of melanoma related transcripts in single round PCR assays. Nonetheless, in the present study, nested PCR for tyrosinase was superior to the combination of single round multimarker RT-PCR analyses in the detection of occult melanoma cells in lymph nodes.

The specificity of RT-PCR analysis needs to be considered carefully before this method can be used routinely. Battyani et al reported positive tyrosinase mRNA expression in five of eight normal lymph nodes, and suggested the presence of normal melanocytes or melanocytic precursors in normal lymph nodes from non-melanoma patients, ${ }^{15}$ although neither we nor others ${ }^{67}$ detected tyrosinase transcripts in any of the normal control lymph nodes by using the same sensitive nested PCR. Furthermore, collections of nevocytes occasionally found in lymph nodes (nodal nevi) ${ }^{16}{ }^{17}$ can be a source of false positivity for MAA RT-PCR. Carson et al found such nodal nevi in melanoma-draining lymph nodes in $22 \%$ of melanoma patients who underwent 
lymphadenectomy. ${ }^{18}$ Thus we examined haematoxylin and eosin and $\mathrm{S} 100$ protein stained sections of all 14 lymph node specimens that were positive for tyrosinase RT-PCR but did not find aggregates of nevus cells in any (data not shown). Therefore we believe that the positive tyrosinase transcripts detected in the lymph nodes from melanoma patients in this study reflected the presence of occult melanoma cells rather than normal melanocytes, melanocytic precursors, or nodal nevi. However, the potential confounder in MAA RT-PCR analysis caused by intranodal benign nevomelanocytic cells should be kept in mind in future studies. The introduction of a novel MAA such as MAGE-3 which may be expressed exclusively on melanoma cells but not on nevomelanocytes ${ }^{19}$ might be of help in overcoming this problem.

The biological significance of occult tumour cells found in the regional lymph nodes of melanoma patients is uncertain. Our statistical analysis comparing the known prognostic factors and recurrence rate between two groups with or without occult micrometastasis showed no difference. However, the statistical power of such a comparison is poor, and our sample was too small for meaningful interpretation. A recent preliminary report suggests that patients with occult melanoma cells detected by tyrosinase RT-PCR in sentinel lymph nodes may have a higher recurrence rate than those whose sentinel nodes were negative. ${ }^{20}$ The same occult tumour cells in the regional lymph nodes have also been reported in various other human cancers. The prognostic significance of such occult micrometastases has been suggested in non-small-cell lung carcinoma, ${ }^{21}$ breast carcinoma, ${ }^{22}{ }^{23}$ and gastric carcinoma. ${ }^{24}$ By contrast, Nasser et al reported that the presence of occult metastases smaller than $0.2 \mathrm{~mm}$, especially those consisting of single cells, did not add useful prognostic information in the patients with node negative breast carcinoma. ${ }^{25}$ Most of the occult tumour cells we found in this study were single cells. The prognostic significance of the occult melanoma cells identified by sensitive immunohistochemical or RT-PCR methods needs to be investigated in future studies, particularly in view of the recent application of sentinel lymph node biopsy for the selection of treatment options.

We are grateful to Professor Jonathan Rees (University of Newcastle Upon Tyne, UK) for critical reading of the manuscript We also thank Yuko Yamada for her excellent technical assistance in immunohistochemistry. This work was partly supported by a grant-in-aid (09770615) for Scientific Research from the Ministry of Education, Science and Culture of Japan to NH. The support of Professor Toshiaki Saida (Shinshu Univer- sity, Japan) by a grant for Cancer Research from the Ministry of Health and Welfare of Japan $(7-40)$ is also appreciated.

1 Drepper H, Biess B, Hofherr B, et al. The prognosis of patients with stage III melanoma. Prospective long-term study of 286 patients of the Fachklinik Hornheide. Cancer 993;71:1239-46.

2 Rusciani L, Petraglia S, Alotto M, et al. Postsurgical adjuvant therapy for melanoma. Evaluation of a 3-year randomized trial with recombinant interferon- $\alpha$ after 3 and 5 years of follow-up. Cancer 1997;79:2354-60

3 Morton DL, Wen DR, Wong JH, et al. Technical details of intraoperative lymphatic mapping for early stage melanoma. Arch Surg 1992;127:392-9.

4 Cochran AJ, Wen DR, Morton DL. Occult tumor cells in the lymph nodes of patients with pathological stage I malignant melanoma. An immunohistological study. Am $\mathcal{F}$ Surg Pathol 1988;12:612-18.

5 Heller R, Becker J, Wasselle J, et al. Detection of submicroscopic lymph node metastases in patients with melanoma. Arch Surg 1991;126:1455-9.

Arch Surg 1991;126:1455-9.
6 Wang X, Heller R, VanVoorhis N, et al. Detection of submicroscopic lymph node metastases with polymerase chain reaction in patients with malignant melanoma. Ann Surg 1994;220:768-74.

7 Van-der-Velde-Zimmermann D, Roijers JF, BouwensRombouts A, et al. Molecular test for the detection of tumor cells in blood and sentinel nodes of melanoma patients. Am f Pathol 1996;149:759-64.

8 Smith B, Selby P, Southgate J, et al. Detection of melanoma cells in peripheral blood by means of reverse transcriptase and polymerase chain reaction. Lancet 1991;338:1227-9.

9 Sarantou T, Chi DD, Garrison DA, et al. Melanomaassociated antigens as messenger RNA detection markers for melanoma. Cancer Res 1997;57:1371-6.

10 Nakajima-Iijima S, Hamada H, Reddy P, et al. Molecular structure of the human cytoplasmic $\beta$-actin gene: interspestructure of the human cytoplasmic $\beta$-actin gene: interspe-
cies homology of sequences in the introns. Proc Natl Acad Sci USA 1985;82:6133-7.

11 Colombari R, Bonetti F, Zamboni G, et al. Distribution of melanoma specific antibody (HMB-45) in benign and malignant melanocytic tumours. An immunohistochemical study on paraffin sections. Virchows Arch 1988;413:17-24.

12 Gown AM, Vogel AM, Hoak D, et al. Monoclonal antibodies specific for melanocytic tumors distinguish subpopulations of melanocytes. Am F Pathol 1986;123:195-203.

13 Bonetti F, Colombari R, Manfrin E, et al. Breast carcinoma with positive results for melanoma marker (HMB-45). HMB-45 immunoreactivity in normal and neoplastic breast. Am f Clin Pathol 1989;92:491-5.

14 Hoon DS, Wang Y, Dale PS, et al. Detection of occult melanoma cells in blood with a multiple-marker polymerase chain reaction assay. F Clin Oncol 1995;13:2109-16.

15 Battyani Z, Xerri L, Hassoun J, et al. Tyrosinase gene expression in human tissues. Pigment Cell Res 1993;6:4005.

16 McCarthy SW, Palmer AA, Bale PM, et al. Nevus cells in lymph nodes. Pathology 1974;6:351-8.

17 Ridolfi RL, Rosen PP, Thaler H. Nevus cell aggregates associated with lymph nodes: estimated frequency and clinical significance. Cancer 1977;39:164-71.

18 Carson KF, Wen DR, Li PX, et al. Nodal nevi and cutaneous melanomas. Am $\mathcal{F}$ Surg Pathol 1996;20:834-40.

9 Hofbauer GFL, Schaefer C, Noppen C, et al. MAGE-3 immunoreactivity in formalin-fixed, paraffin-embedded primary and metastatic melanoma. Am F Pathol 1997;151: 1549-53.

20 Reintgen DS, Conrad AJ. Detection of occult melanoma cells in sentinel lymph nodes and blood. Semin Oncol 1997; 24: $111-15$.

21 Chen ZL, Perez S, Holmes EC, et al. Frequency and distribution of occult micrometastases in lymph nodes of bution of occult micrometastases in lymph nodes of
patients with non-small-cell lung carcinoma. $\mathcal{F}$ Natl Cancer Inst 1993;85:493-8.

22 Trojani M, Mascarel ID, Bonichon F, et al. Micrometastases to axillary lymph nodes from carcinoma of breast: detection by immunohistochemistry and prognostic significance. Br f Cancer 1987;55:303-6.

23 Hayashi N, Ito I, Yanagisawa A, et al. Genetic diagnosis of lymph-node metastasis in colorectal cancer. Lancet 1995; 345:1257-9.

24 Ishida K, Katsuyama T, Sugiyama A, et al. Immunohistochemical evaluation of lymph node micrometastases from gastric carcinomas. Cancer 1997;79:1069-76.

25 Nasser I, Lee A, Bosari S, et al. Occult axillary lymph node metastases in "node negative" breast carcinoma. Hum Pathol 1993;24:950-7. 